\title{
Smart cities platform: ensuring artificial intelligence makes a right decision
}

\section{Opinion}

Smart Cities Platform uses historical data to identify patterns and prevent the unforeseen events by prediction. Cities around the world are aspiring to provide superior quality of life to their citizens while optimizing their cumulative resource footprint. Artificial Intelligence plays an important role in making safer cities, transport smarter and urban planning optimal. The smart city offers a rich avenue for applying AI algorithms like machine learning, planning, scheduling, semantics, knowledge representation, data integration, logic, trust and agents.

AI Platform tracks citizen's habits, activities, and behavioral characteristics. Data and products can be personalized to meet and anticipate each user's unique and changing needs. Each citizen will have one's own digital personal assistant. Artificial intelligence can help governments handle their regulations monitoring by creating a natural language processing system to read through the legalities of regulations and reassemble the words into a set of computerunderstandable rules. IoT, AI, VR, AR, and bots technologies are changing the way data is created, collected, interpreted, and communicated.

As governments begin to rely more on data-driven Artificial Intelligence applications, the new applications lead to new issues, security, and privacy concerns. Each government department also needs to have a transparent system for total audit ability so one can see who did what, and when. Deep Learning platform users should identify erroneous or incomplete data to avoid misleading decisions. The new AI applications introduce a number of business, security and privacy issues which will have to be addressed.
Volume 3 Issue I - 2016

Bhagvan Kommadi

Architect Corner, Stanford University Graduate School of Business, India

Correspondence: Bhagvan Kommadi, Architect Corner, Stanford University Graduate School of Business, India, Email bhagvank@architectcorner.com

Received: October 27, 2016 | Published: November 10, 2016

It will be important to ensure that these intelligent applications are developed in a way that they will provide the desired benefit and that the user can trust the advice and services provided. It will be important to be able to detect and isolate infected or malicious AI programs immediately, and develop the effective policy and laws for governing their development and use, so that personal information is safeguarded and not misused.

\section{Acknowledgments}

None.

\section{Conflicts of interest}

None. 